 \\ X-Ray Fluorescence Microscopy DE91 004799
}

\author{
M. L. Rivers and S. R. Sutton \\ Department of the Geophysical Sciences \\ The University of Chicago, Chicago, Illinois 60637
}

\author{
K. W. Jones \\ Department of Applied Science \\ Brookhaven National Laboratory, Upton, New York 11973
}

\section{DISCLAIMER}

\begin{abstract}
This report was prepared as an account of work sponsored by an agency of the United States Government. Neither the United States Government nor any agency thereof, nor any of their employees, makes any warranty, express or implied, or assumes any legal liability or responsibility for the accuracy, completeness, or usefulness of any information, apparatus, product, or process disclosed, or represents that its use would not infringe privately owned rights. Reference herein to any specific commercial product, process, or service by trade name, trademark, manufacturer, or otherwise does not necessarily constitut: or imply its endorsement, recommendation, or favoring by the United States Government or any agency thereof. The views and opinions of authors expressed herein do not necessarily state or reflect those of the United States Government or any agency thereof.
\end{abstract}

\section{Presented at}

X-Ray Microscopy 1990

King's College, London, United Kingdom

September 3-7, 1990

\section{MASTER}

By acceptance of this article, the publisher and/or recipient acknowledges the US Government's right to retain a nonexclusive, royalty-free license in and to any copyright covering this paper. 


\title{
X-ray Fluorescence Microscopy
}

\author{
Mark L. Rivers, Stephen R. Sutton \\ Department of the Geophysical Sciences, The University of Chicago, \\ 5734 S. Ellis Avenue, Chicago, IL 60637 \\ Keith W. Jones \\ Department of Applied Science, Brookhaven National Laboratory, \\ Building 815, Upton, NY 11973
}

\begin{abstract}
Synchrotron $x$-ray flucsescence microscopy is used to quantitatively measure and image the distribution of trace elements in biological, geological and materials science specimens. The design and performance of the $x$-ray fluorescence (XRF) microprobe at the NSLS are discussed and compared with other XRF microprobe designs. An example of a trace element image obtained with this instrument is presented.
\end{abstract}

\section{INTRODUCTION}

The micro-distribution of minor and trace elements is of great interest in fields such as geochemistry, biology and material science. The synchrotron $x$-ray fluorescence microprobe (SXFM) provides a technique to quantitatively measure trace element compositions at individual points and to construct two dimensional images of trace element compositions. A SXFM has been in operation at the NSLS for nearly five years $[1,2]$. This paper will present some of the recent improvements and results wich have been obtained with this instrument.

The SXFM at the NSLS is portable and can be operated on a number of beamlines. It is run in a nearly dedicated mode on beamline $X-26 \mathrm{~A}$, which is a white light bending magnet beamline with a hut $\mathrm{h}$ located only $9 \mathrm{~m}$ from the source point. This beamline has an 8:1 ellipsoidal mirror installed [3]. The SXFM can also operate on beamline $\mathrm{X}-26 \mathrm{C}$, which is located $20 \mathrm{~m}$ from the source and has a $1: 1$ cylindrical focussing mirror installed. The performances of the $8: 1$ and $1: 1$ mirrors are described below. When the SXFM is used as a collimated microprobe, without the focussing mirrors, the X-26A beamline provides a factor of 5 gain in tux over $\mathrm{X}-26 \mathrm{C}$, simply by virtue of being closer to the source. Finally, the SXFM can also be operated on the X-17B1 superconducting wiggler beamline. This beamline has a critical energy of $20 \mathrm{keV}$, which is four times that of the bending magnet. This device provides usable flux to beyond $100 \mathrm{keV}$, making it suitable for the $\mathrm{K}$ shell excitation of any element.

There are a number of techniques in use for producing hard $x$-ray microbeams for XRF work. Among them are grazing incidence mirrors [4], Kirkpatrick-Baez multilayercoated mirrors [5] and ellipsoidally bent Si crystals [6]. While we have installed grasing incidence mirrors on our beamlines, the SXFM is operated most of the time as a collimated, white-light microprobe, without any focussing optics. The reason for this is that the performance on a low-emittance storage ring such as the NSLS, on a beamline 
close to the source (such as $\mathrm{X}-26 \mathrm{~A}$ ) is for most purposes superior to that which has been ohtained with more complex focussing microprobes. The performance criteria which we consider important include spatial resolution, sensitivity, range of elements which can be excited, and ease of use. As an example, Figure 1 compares the X-26A collimated microprobe with the Kirkpatrick-Baez (K-B) multilayer microprobe of Giauque et. al (5). Both microprobes have beam sizes of $5.8 \mu \mathrm{m}$ and were run on an NSLS bending magnet. The figure shows the fluorescent signal from a thin standard as a function of energy. The (K-B) microprobe is monochromatic with an energy of $10 \mathrm{keV}$ and a $10 \%$ bandwidth, hile the X-26A microprobe uses white light, with significant flux from 3 to $25 \mathrm{keV}$. The K-B microprobe produces a better signal for $\mathrm{Zn}$ and $\mathrm{Cu}$, whose $\mathrm{K}$ absorption edges are close below $10 \mathrm{keV}$. However, the X-26A microprobe has better sensitivity for both lighter and heavier elements. In particular the X26-A microprobe is capable of accessing elem.'nts such as $R b, S_{r}, Y$, and $\mathrm{Zr}$ which are very important in geochemical studies.

\section{POLARIZATION}

High flux and low backgrounds are both required to produce a SXFM with good elemental sensitivity. The principal source of background in the SXFM is coherent and incoherent scatter. In the case of the white-light microprobe this is obvious: the incident beam contains photons whose energy (with or without Compton shifting) is equal to that of th. Euorescent radiation one is trying to detect. With monochromatic radiation the effect is more subtle: the scattered photons will have an eliergy greater than that of the fluorescent peaks. However, the background in a $\mathrm{Si}(\mathrm{Li})$ detector is controlled by incomplete charge collection of these photons, so the scattering still determines the background signal. This scattered background can be minimized by taking advantage of the linear polarisation of the radiation from bending magnet and wiggler sources. By placing the detector in the plane of polarization at $90^{\circ}$ to the incident beam the intensity of scattered ohotons is minimized. For these experiments the degree of linear polarisation is critical. The polarisation is at a maximum in the vertical center of the radiation profile. At the X-26A beamline we regularly measure the ratio of the scattered background to the $\mathrm{Sr} \mathrm{K \alpha}$ fluorescence peak on a glass standard in order to position the experiment exactly in the vertical center of the beam. This ratio changes by a factor of 4 when the experiment is moved $\pm 0.6 \mathrm{~mm}$ vertically (Figure 2 ). Since the degree of linear polarization from a wiggler source should be better than that from a bending magnet (due to cancellation from opposite poles in the wiggler), we had expected this ratio to be better when this standard was tun on X-17B1 than on X-26A. In fact the ratio was 2.9 times pooret and was quite insensitive to the vertical position (Figure 2). In an attempt to understand these observations we have done Monte Carlo calculations to predict the linear polarization of a dipole suurce with a finite emittance beam. For the predictions one needs to specify the following items: the ratio of the energy to the critical energy energy $E / E_{c}$, the vertical source emittance $\epsilon_{y}$, the vertical beta function $\beta_{y}$, distance from the source, and the machine energy. The values we used were for the NSLS $x$-ray ring $\left(2.52 \mathrm{GeV}, \epsilon_{y}=10^{-9} \mathrm{~m}\right.$ tad), at an $x$-ray energy of $17 \mathrm{keV}$. The results for the $\mathrm{X}-26 \mathrm{~A}$ dipole source $\left(\beta_{y}=15 \mathrm{~m}\right.$, distance $\left.=9 \mathrm{~m}, E / E_{c}=3.4\right)$ and the X-17 B1 beamline $\left(\beta_{y}=.33 \mathrm{~m}\right.$, distance $\left.=27 \mathrm{~m}, E / E_{c}=.9\right)$ are shown in Figure 3 . The results are quite surprising. The wiggler source has much poorer polarization 
(96.4\%) than the X-26A dipole (99.5\%). The difference is due entirely to the difference in $\beta_{y}$ at the two locations, not to the differences in the critical energy of the sources. The very small beta function at the X-17 source point leads to an electron beam which is extremely small but whose divergence is much greater than the intrinsic vertical opening angle of the synchrotron tadiation. This leads to a "smearing out ${ }^{n}$ of the radiation distribution and a reduction in the peak on-axis polarization. The predicted polarization of the $X-17$ radiation is 7.3 times poorer than that at $X-26$. The observed increase in the scattered intensity, however, is only a factor of 2.9 . The difference is probably due to the fact that we have modeled the X-17 wiggler as a dipole source and neglected the cancelation from the opposite poles of the wiggler.

\section{COLLIMATION}

Collimators for use in SXFM must be capable of producing as small a beam as possible with minimal scattered intensity. When used on a $x$-ray synchrotron source such as the NSLS they must also be able to block the high energy (>30 keV) photons.

The collimators which we use are made from crossed pairs of Ta slits. The slits are $1 \mathrm{~mm}$ thick with edges polished to better than $1 \mu \mathrm{m}$. Opposing slits are held apart using $8 \mu \mathrm{m}$ Kapton film at the edges. In most areas the $\mathrm{Ta}$ is thus $2 \mathrm{~mm}$ thick, which is sufficient to stop the high-energy radiation from an NSLS bending magnet. In fact we hare successfully used these same collimators on the $\mathrm{K}-17 \mathrm{~B}$ superconducting wiggler beamline, asing beam energies of $60-80 \mathrm{keV}$.

The collimator is mounted on a stepper motor driven stage with $X-Y$ translation capability and $\theta-\phi$ rotation capability. The rotational capability is necessary, since the collimator has a very large aspect ratio, greater than 120:1. By rotating about the vertical and horizontal axes one can align the collimator so that it is "wide open", i.e. pointing directly at the source. However, it is also possible to deliberately misalign the collimator using these rotations. This decreases the effective sise of the collimator and can be used to produce beams much smaller than $8 \mu \mathrm{m}$.

On X-26A we routinely use the collimators to produce beam sizes of $6 \mu \mathrm{m}$ in the horizontal and vertical directions (Figure 4) with the SXFM. In the SXFM the specimen is mounted at $45^{\circ}$ to the incident beam and the minimum specimen-collimator distance is $30 \mathrm{~mm}$. Since the distance from the source is $9 \mathrm{~m}$ and the horizontal source size is about $1 \mathrm{~mm}$ (FWEM), the minimum spot size which we can produce is $3 \mu \mathrm{m}$. In microtomography experiments the collimator can be placed within $2 \mathrm{~mm}$ of the sample. In these experiments minimum beam sises of $1 \mu \mathrm{m}$ have been obtained.

When using this collimator system in air with the white-light SXFM we have found that the spectrum of the $x$-rays transmitted thought the collimator grows gradually harder with time, i.e. there is a loss of the low energy $x$-rays. Disassembling the slits after a few weeks of use reveals them to be covered with a thin film. The beam passes through this growing film and the low energy photons are absorbed. Since the slits were used with white beam in air we had thought that this film was probably Ta oxide produced by the interaction of ozone and the Ta metal. However, examination with electron and Auger microprobes revealed that the film was predominately carbon. Apparently even in air the $x$-ray beam is producing carbonaceous contamination. Flowing He though the collimator assembly has eliminated or greatly reduced this contamination problem. 


\section{FOCUSSING OPTICS}

We have measured the performances of the $1: 1$ and $8: 1$ mirtors installed on the $\mathrm{X}-26 \mathrm{C}$ and X-26A beamlines respectively. The $1: 1$ mirror accepts $4 \mathrm{mrad}$ in the horirontal direction and $0.2 \mathrm{mrad}$ in the vertical at a grasing angle of $5 \mathrm{mrad}$. The measured focal spot has a FWHM of $600-700 \mu \mathrm{m}$ in both the vertical and horizontal directions. It is thus focussing $1: 1$ in the horizontal and is doing some focussing in the vertical, implying that it is actually toroidal in shape. The increase in int nsity is a factor of 100 over the unfocussed white beam over the energy range of $5.12 \mathrm{keV}$ (Figure 5), which is very close to the theoretical performance. The $8: 1$ ellipsoidal mirror accepts $0.5 \mathrm{mrad}$ in the horizontal. It produces a measured focal spot of $125 \mu \mathrm{m}$ (H) by $350 \mu \mathrm{m}$ (V). It thus focuses $8: 1$ in the horisontal but only $1: 1$ in the vertical. The increase in intensity is only a factor of 30 over the unfocussed radiation, whereas the theoretically expected value is close to 2000 . The problems with the surface roughness and slope errors of this mirror have been discussed elsewhere [3].

\section{IMAGING}

An example of the type of imaging which is performed with the SXFM at X-26A is shown in Figure 6. This is a scan of calcite $\left(\mathrm{CaCO}_{3}\right)$ from a fracture in a coal seam. The image was collected by setting software selected regions-of-interest around each peak and collecting the net counts above background during each 5 second pixel. The $\mathrm{Ca}$ concentration is quite uniform. Howerer the $\mathrm{Fe}, \mathrm{Mn}$ and $\mathrm{Sr}$ distributions show that the calcite was formed by infiltration of several different fluids which had different trace element concentrations.

\section{ACKNOWLEDGMENTS}

This research was supported by NSF EAR89-15699, NASA NAG9-106, DoE DE-AC0276CH00016. Dr. Allan Kolker kindly provided the calcite image.

\section{REFERENCES}

1. M. L. Rivers in X.ray Microscopy II, D. Sayer, M. Howells, J. Kirz, H. Rarback eds. (Springer-Verlag, New York) 233 (1988)

2. F. Q. Lu, J. V. Smith, S. R. Sutton, M. L. Rivers, A. M. Davis: Chem. Geol. 75123 (1989)

3. K. W. Jones, P. E. Takacs, J. B. Hastings, J. M. Casstevens, C. D. Pionke: Soc. Photo-Optical Engineers, 749, 37 (1987)

4. S. Hayakawa, A. Lida, S. Aoki, Y. Gohshi: Rev. Sci. Instrum. 602452 (1989)

5. R. D. Giauque, A. C. Thompson, J. H. Undermood, Y. Wu, K. W. Jones, M. L. Rivers: Anal. Chem., 60, 855 (1988)

6. F. Van Langevelde, G. B. J. Tros, D. K. Bowen, R. D. Vis: Nucl. Instr. Meth. B49 $544(1990)$ 


\section{FIGURE CAPTIONS}

\section{Figure 1.}

Comparison of the fluorescence signal from a thin standard using a Kirkpatrick-Baez multilayer microprobe on beamline X-26C $[5]$ and the X-26A collimated microprobe. The vertical su-e is fluorescent $x$-ray counts/second/picogram/100 mA beam current $/ 0.0018$ steradians detector solid angle. Beam sise for both instruments is about $5 \mu \mathrm{m}$.

\section{Figure 2.}

The ratio of the scattered background to the fluorescent peak (Sr $\mathrm{K}_{\alpha}$ ) measured as a function of vertical beam position on bending magnet beamaline X-26A and superconducting wiggler beamline $X-17 \mathrm{~B} 1$. The vertical units are $\gamma \psi$ where $\gamma$ is equal to $195 T \times E_{G e V}$ and $\psi$ is the angle from the center of the radiation.

\section{Figure 3.}

The predicted polarization of the radiation from the X-26A bending magnet and the $\mathrm{X}-17 \mathrm{~B} 1$ superconducting wiggler. The prediction was done using a Monte-Carlo simulation which includes the effects of the vertical beam emittance and the vertical beta function.

\section{Figure 4.}

Vertical scan across a grid of $0.3 \mu \mathrm{m}$ thick Au deposited on $0.1 \mu \mathrm{m}$ thick SiN. The signal measured is the $A n L \alpha$ fluorescence peak. The beam size is $6 \mu \mathrm{m}$, determined from the $10-90 \%$ points. The signal from the scattered "halo" is less than $1 \%$ when the beam is $10 \mu \mathrm{m}$ away from the Au edge.

\section{Figure 5.}

The intensities of the focussed and unfocussed beams at X-26C as a function of energy. The intensities were measured with a Si(Li) detector at an angle of $45^{\circ}$ to the incident beam. The measurements were made by scanning a $50 \mu \mathrm{m}$ pinhole through the beam to find the peak intensity of the radiation.

\section{Pigure 0 .}

Scanning images of calcite from a fracture in a coal seam. The scan dimensions are 2 $\mathrm{mm}$ (horizontal) by $1 \mathrm{~mm}$ (vertical). The image was collected with a $10 \mu \mathrm{m}$ beam but with $25 \mu \mathrm{m}$ pixels in order to scan a large area in a reasonable time. The time per pixel was 5 seconds. 


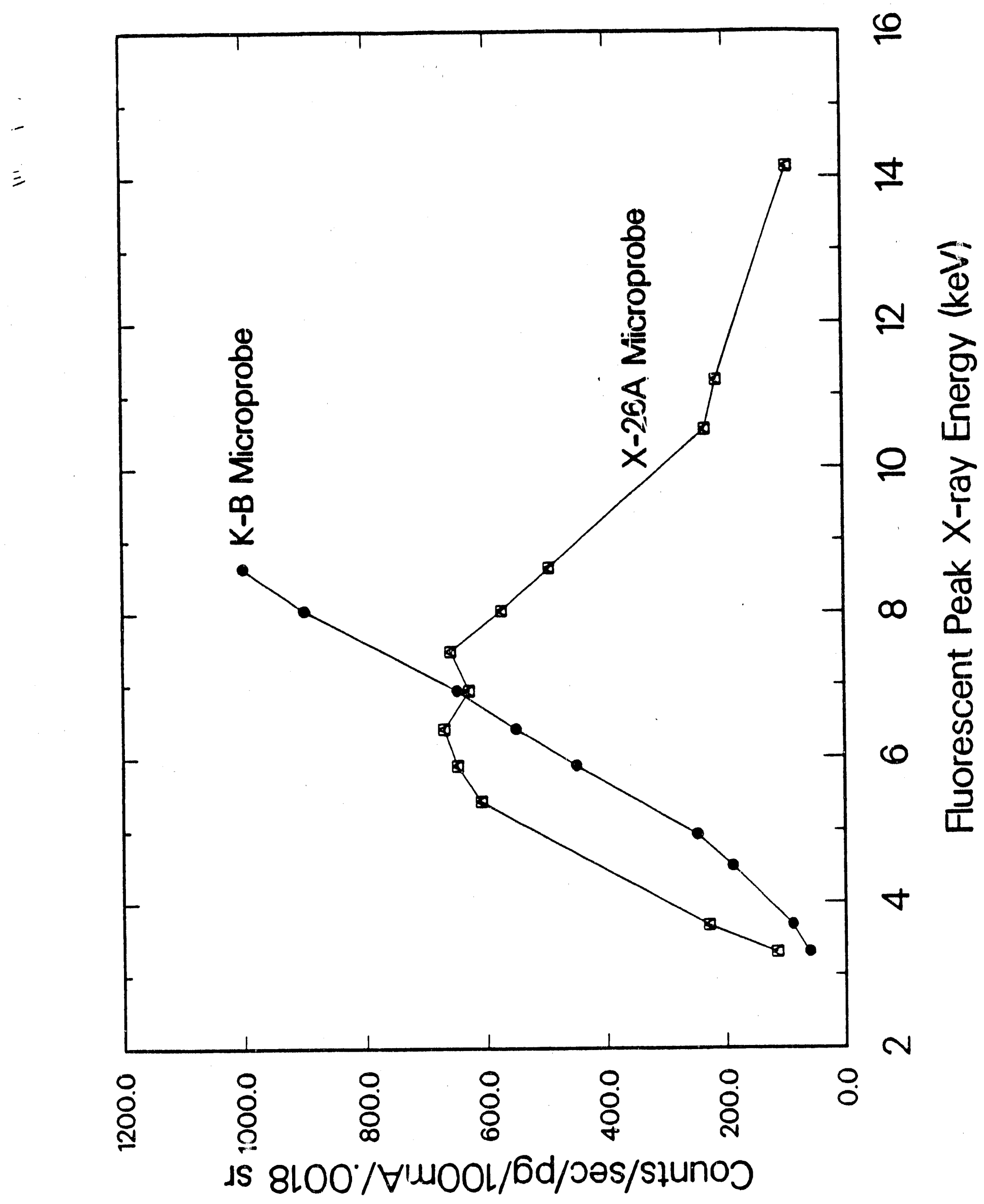




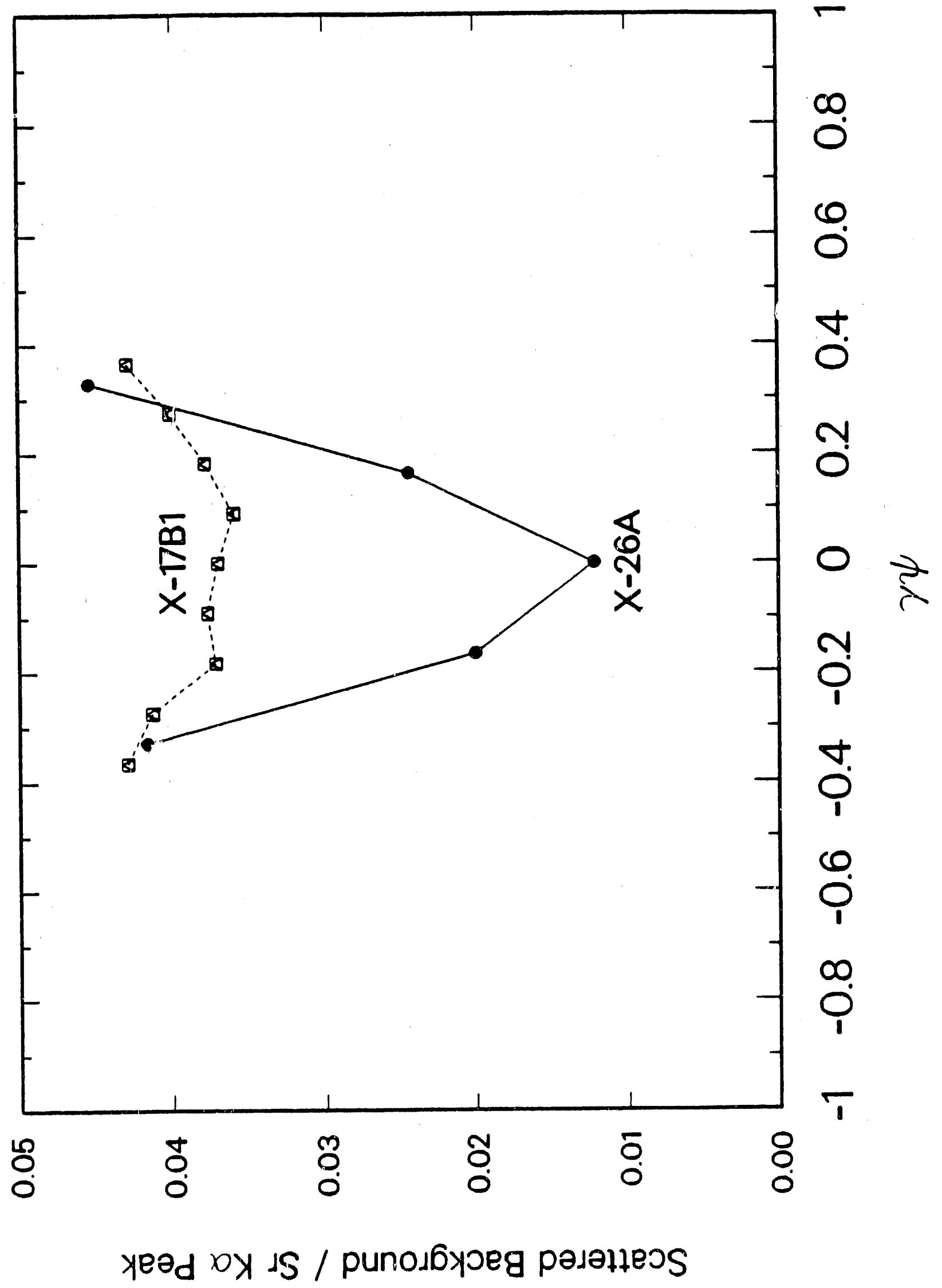



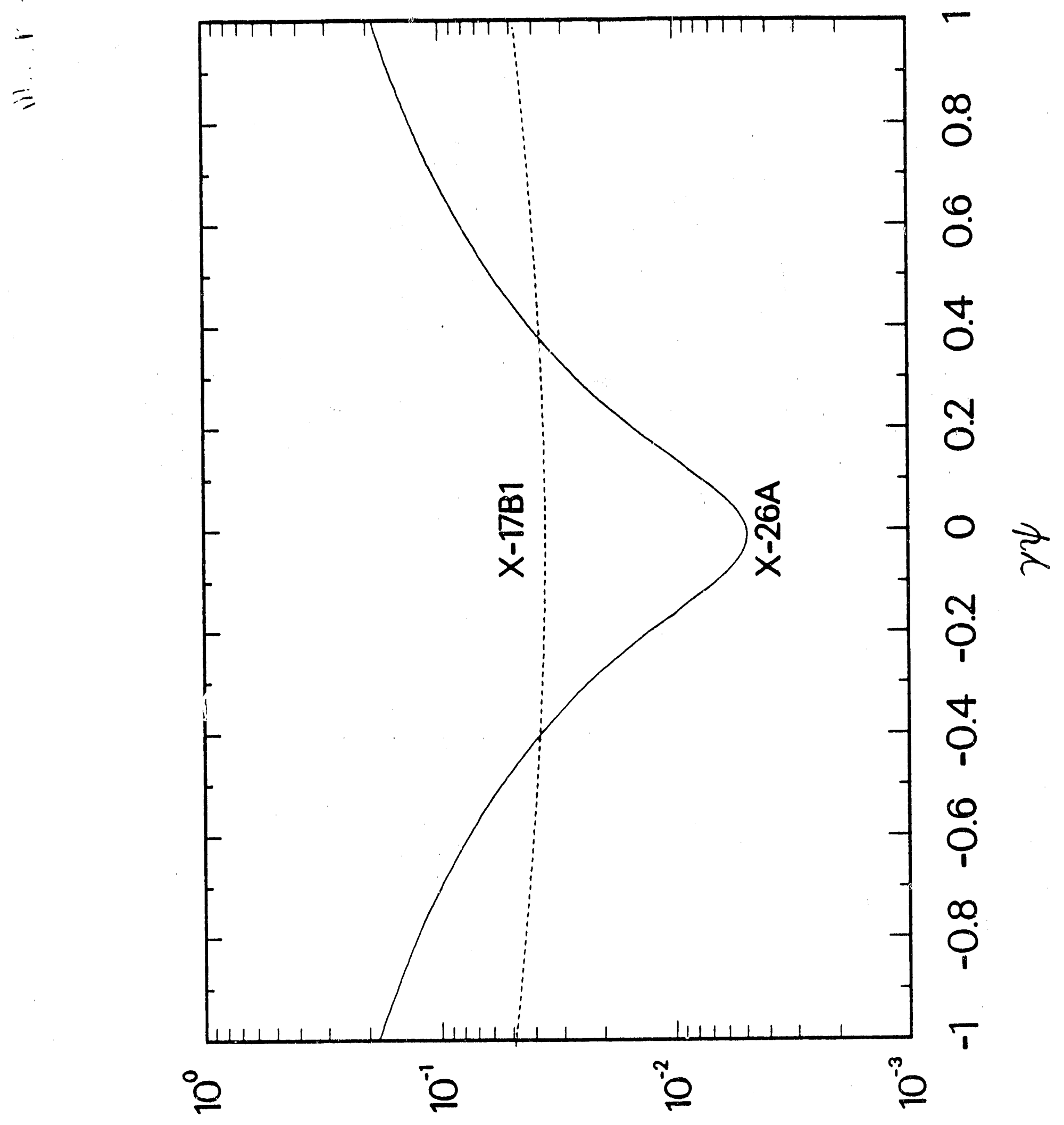

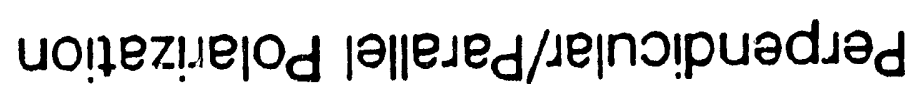




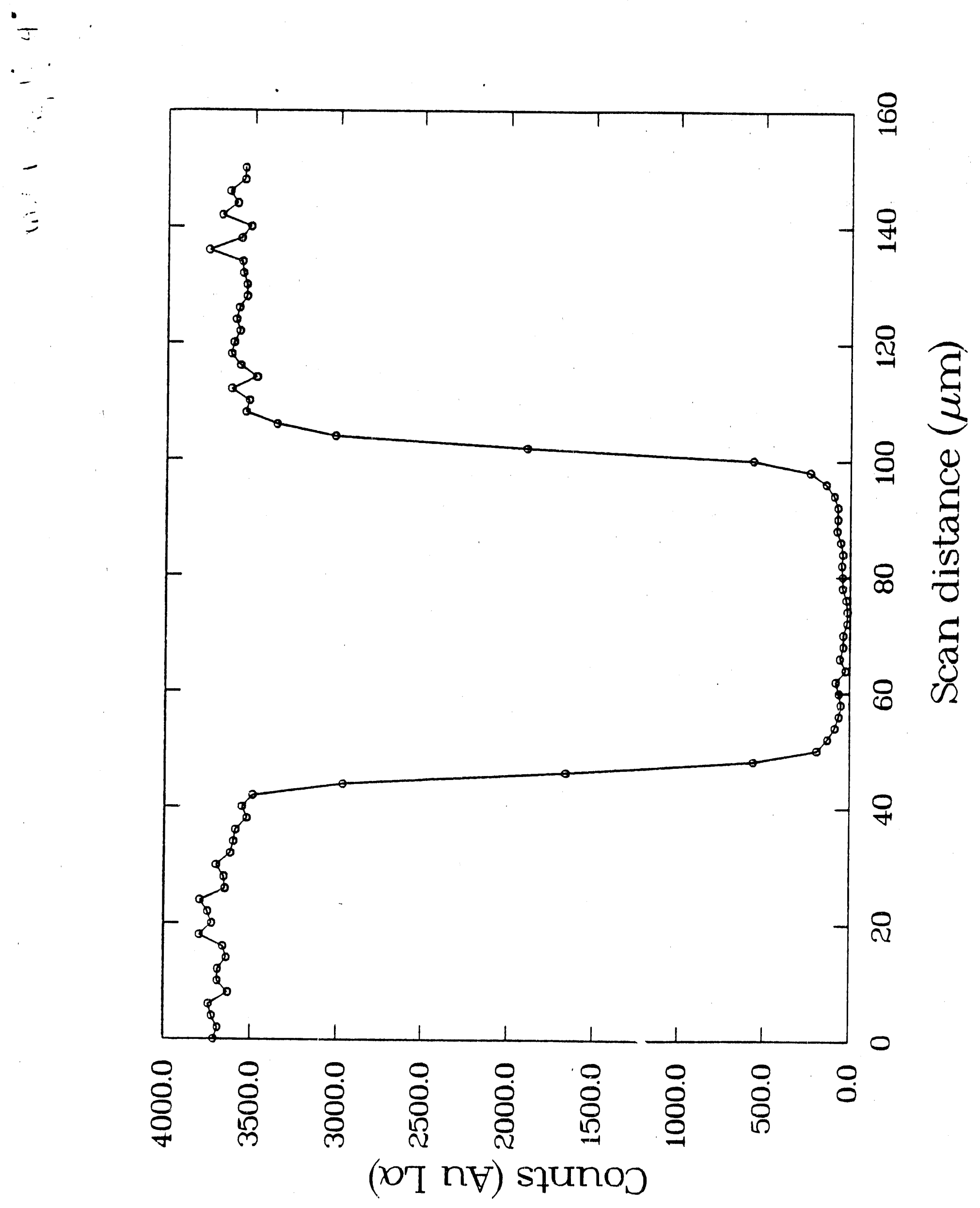




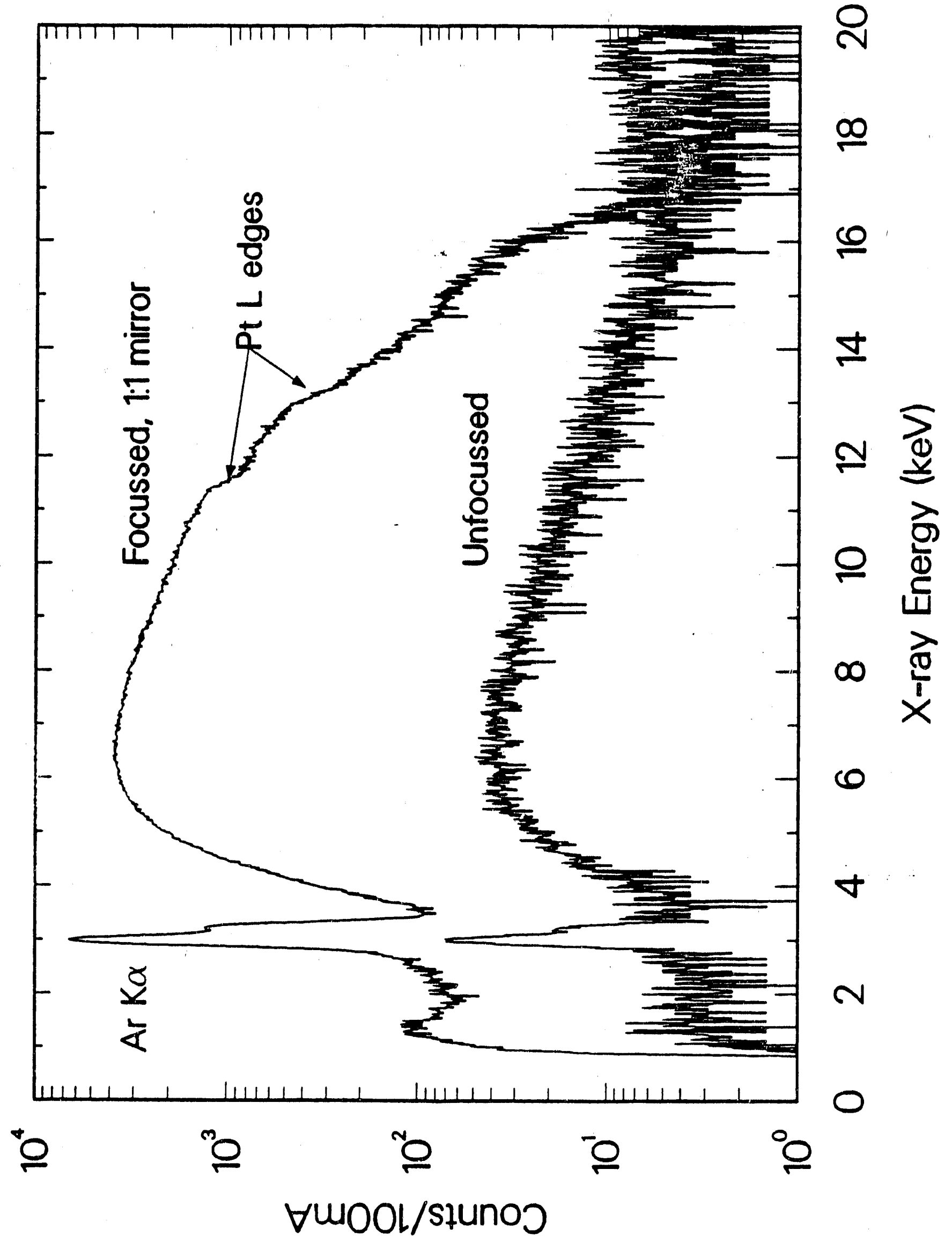



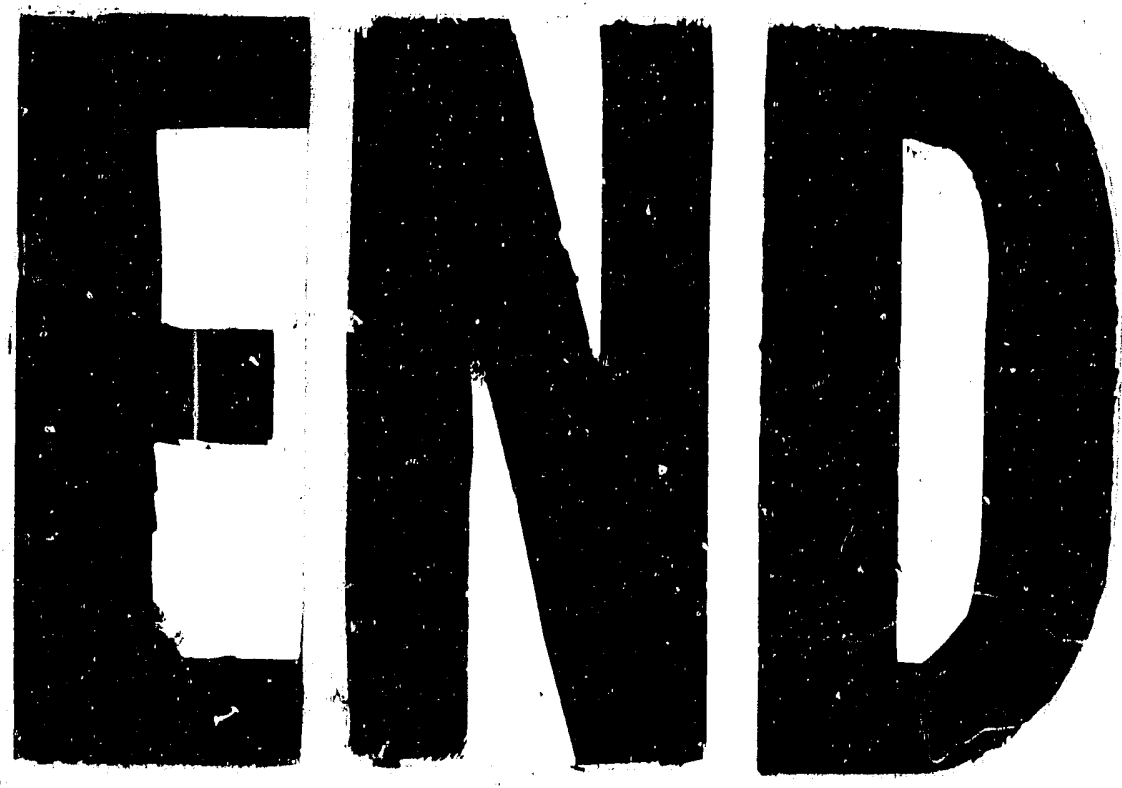

5
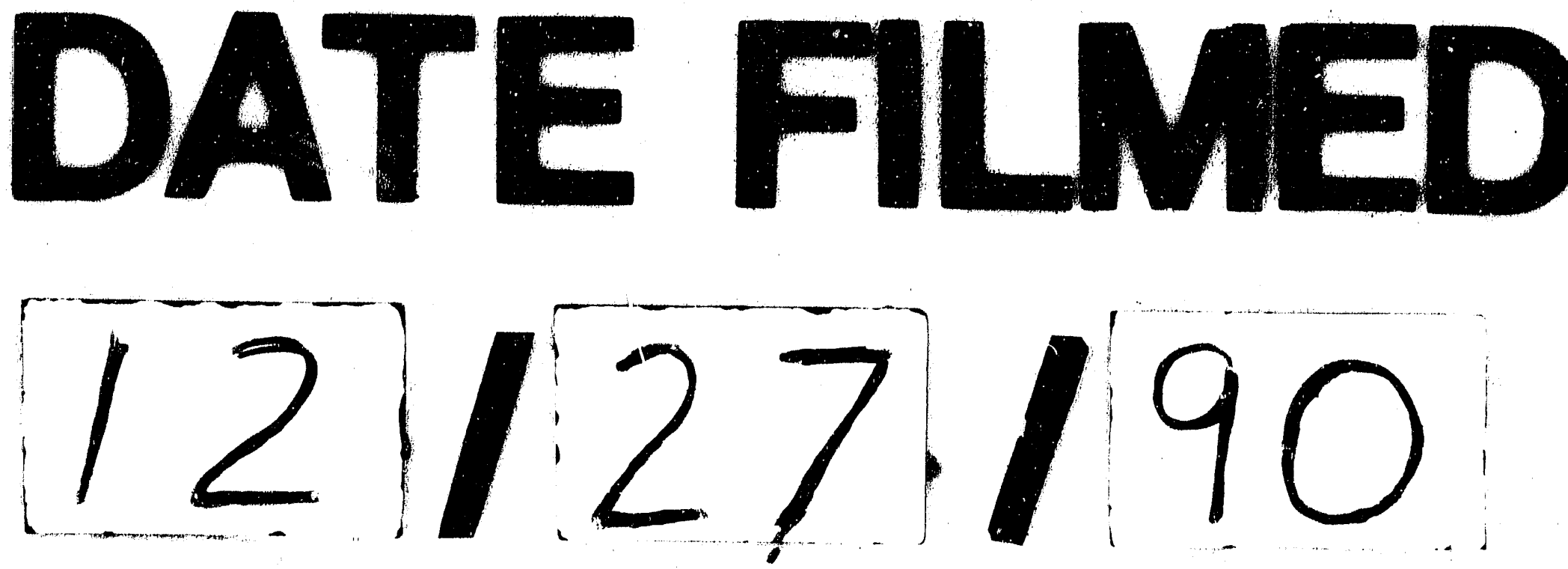